\title{
SOME INFINITE FACTOR GROUPS OF BURNSIDE GROUPS
}

\author{
Dedicated to the memory of Hanna Neumann
}

\author{
J. L. BRITTON
}

(Recieved 3 July 1972)

Communicated by M. F. Newman

Let $B_{d}(e)$ denote the Burnside group with $d \geqq 2$ generators $a_{1}, a_{2}, \cdots, a$, and exponent $e>0$, i.e., the free group of rank $d$ of the Burnside variety of exponent $e$. It is known that $B_{d}(e)$ is infinite for all sufficiently large odd values of $e$; cf. Novikov and Adyan [3] or Britton [1]. In particular $B_{d}\left(p^{k}\right)$, where $p$ is an odd prime, is infinite for all sufficiently large $k$. It is not known whether or not $B_{d}\left(2^{k}\right)$ is infinite for all sufficiently large $k$; infiniteness would imply that $B_{d}(n)$ is infinite for all sufficiently large $n$, as has been conjectured by Novikov [2].

Now let $J_{2}\left(p^{k}\right)$, where $p$ is prime, be the factor group of $B_{2}\left(p^{k}\right)$ obtained by adding the defining relations $a_{1}^{p}=1, a_{2}^{p}=1$.

THEOREM 1. (i) If $p$ is odd, $J_{2}\left(p^{k}\right)$ is infinite for all sufficiently large $k$.

(ii) If $p=2, J_{2}\left(p^{k}\right)$ is finite for all $k$.

The proof of (ii) is trivial since the relations $a_{1}^{2}=a_{2}^{2}=\left(a_{1} a_{2}\right)^{2^{k}}=1$ define a dihedral group of order $2^{k+1}$. (i) is a special case of Theorem 2 below.

Let $\Pi=G_{1} * G_{2} * \cdots * G_{r}$ be a free product of $r \geqq 2$ finite groups of odd otder. An element $X \in \Pi, X \neq 1$, whose normal form is

$$
x_{1} x_{2} \cdots x_{n} \quad\left(x_{i} \in G_{f(i)}, i=1,2, \cdots, n\right)
$$

is called externally reduced if $n \geqq 2$ and $f(1) \neq f(n)$; let $J_{0}$ be the set of all such elements. Let $\Gamma^{e}(S)$, where $e>0$ and $S$ is any subset of $J_{0}$, be the group obtained from $\Pi$ by adding the defining relation

$$
X^{e}=1 \quad(X \in S)
$$

THEOREM 2. $\Gamma^{e}\left(J_{0}\right)$, hence $\Gamma^{e}(S)$, is infinite for all sufficiently large odd values of $e$.

Proof. This follows from the author's paper [1] in view of the second sentence of Section 2. of Chapter II. 
Note that $\Gamma^{e}\left(J_{0}\right)$ is finitely generated and has a (non-zero) exponent Of course, if all $G_{t}$ are cyclic of order $e$ then $\Gamma^{e}\left(J_{0}\right)$ is $B_{r}(e)$.

To prove (i) of Theorem 1 take $r=2, G_{i}$ cyclic of order $p(i=1,2), e=p^{k}$; then $\Gamma^{e}\left(J_{0}\right)$ is $J_{2}\left(p^{k}\right)$.

\section{References}

[1] J. L. Britton, The existence of infinite Burnside groups, article in: Word problems Studies in Logic and the Foundations of Mathematics (ed. by Boone, W. W., Cannonito, F. B., Lyndon, R. C.). Amsterdam. North-Holland). (1972).

[2] P. S. Novikov, 'On periodic groups', (Russian) Dokl. Akad. Nauk SSSR (1959) 127 749-752,

[3] P. S. Novikov, Adjan, S. I. 'On infinite periodic groups' (Russian) Izv. Akad. Nauk. SSSR, Ser. Mat. (1968) 212-244, 251-524, 709-731.

The University

Canterbury, Kent England 